This item was submitted to Loughborough's Research Repository by the author.

Items in Figshare are protected by copyright, with all rights reserved, unless otherwise indicated.

\title{
Critical fields and devil's staircase in superconducting ladders
}

PLEASE CITE THE PUBLISHED VERSION

LICENCE

CC BY-NC-ND 4.0

\section{REPOSITORY RECORD}

Giles, R.T., and F.V. Kusmartsev. 2019. "Critical Fields and Devil's Staircase in Superconducting Ladders". figshare. https://hdl.handle.net/2134/1235. 


\title{
Critical fields and devil's staircase in superconducting ladders
}

\author{
Richard T Giles and Feodor V Kusmartsev \\ Department of Physics, Loughborough University, Loughborough, LE11 3TU, U.K.
}

(March 6, 2006)

We have determined the ground state for both a ladder array of Josephson junctions and a ladder of thin superconducting wires. We find that the repulsive interaction between vortices falls off exponentially with separation. The fact that the interaction is short-range leads to novel phenomena. The ground state vortex density exhibits a complete devil's staircase as the applied magnetic field is increased, each step producing a pair of metal-insulator transitions. The critical fields in the staircase are all calculated analytically and depend only on the connectivity of the ladder and the area of the elementary plaquette. In particular the normal square ladder contains no vortices at all until the flux per plaquette reaches $\frac{\Phi_{0}}{2 \sqrt{3}}$.

It is now relatively easy to fabricate complex structures containing many Josephson junctions. Two-dimensional arrays have long been of interest. In a transverse magnetic field, both square [1] and triangular [2, 20 2D arrays show very rich structure in resistance, impedance and inverse sheet inductance measurements when the flux per plaquette is close to a simple rational fraction (at which commensurate states form). Such arrays appear to be well described by a frustrated XY model [5 13] although special attention has only been given [5.9, 14. to the simplest rational flux values: $f=\frac{1}{2}, \frac{1}{3}$ and $\frac{2}{5}$. Vortex dynamics in 2D arrays display many anomalous features [15, 16. Interesting self-inductance [17, 18] and charging 19. effects are also observed.

Analogous phenomena are observed in superconducting weakly coupled wire networks made in thin amorphous niobium silicon films [20,21]. Furthermore MonteCarlo calculations 22 on a Coulomb gas model of a $2 \mathrm{D}$ network have indicated the existence of two melting transitions in the vortex sub-system.

Recently there has been much interest in ladder arrays of Josephson junctions. Experimental work [23] on wide ladders has found a rich structure in the dependence of the longitudinal resistance on transverse magnetic field and this has been interpreted in terms of metal-insulator transitions. In numerical calculations 24 it has been noticed that the normal square Josephson ladder exhibits a critical field below which the ground state contains no vortices while Monte-Carlo simulations have shown, for the case $f=\frac{1}{2}$, that the ladder belongs 25 to the universality class of the Ising model.

A quasi-classical analytical method for investigating
Josephson networks has been successfully tested 26] against full Monte-Carlo simulations. In this paper we use the same method to completely determine the ground state, as a function of applied magnetic field, of both the Josephson ladder shown in Fig. 1a and of the analogous superconducting wire network shown in Fig. 1b. We find that the vortex-vortex interaction falls off exponentially with separation, in contrast to the long-range logarithmic interaction between vortices in $2 \mathrm{D}$ and $3 \mathrm{D}$ arrays, and that at low temperatures the ladder is described by an Ising model. The short-range nature of the interaction gives rise to a geometry dependent critical field $H_{g}$ which depends solely on the geometrical structure of the ladder and plays the same role as $H_{c 1}$ in bulk superconductors. Above $H_{g}$ the vortex density exhibits a complete devil's staircase as the applied field is increased. To our knowledge, this is the first example of a real physical system exhibiting a non-trivial complete devil's staircase.

First we study the Josephson ladder and then show that the analogous ladder of superconducting wires behaves in the same way. A network of Josephson junctions can be described by the XY-model [27]:

$$
H=\frac{I_{c} \Phi_{0}}{2 \pi} \sum_{i}\left(1-\cos \phi_{i}\right)
$$

where the sum is over all junctions, $I_{c}$ is the critical current and $\Phi_{0}=\frac{h}{2 e}$ is the flux quantum. The gaugeinvariant phase difference $\phi_{i}$ across the $i$ th junction is defined by

$$
\phi_{i}=\Delta \theta_{i}-\frac{2 \pi}{\Phi_{0}} \int_{i} \mathbf{A} \cdot \mathrm{d} \mathbf{l}
$$

where $\Delta \theta_{i}$ is the phase difference and the line integral of the vector potential $\mathbf{A}$ is taken across the junction. The current through the $i$ th junction is given by

$$
I_{i}=I_{c} \sin \phi_{i}
$$

Consider a ladder (Fig. 1a), which we call the Josephson ladder, consisting of a linear series of identical plaquettes. Let there be $2 u$ unshared junctions in each plaquette and $c$ junctions in each region which is in common between two plaquettes. Let the applied magnetic flux per plaquette be $f \Phi_{0}$. The sum of the gauge invariant phase differences around any plaquette is then quantised according to:

$$
\sum_{\text {plaquette } j} \frac{\phi_{i}}{2 \pi}=n_{j}-f-\frac{1}{2(u+c) I_{\Phi_{0}}} \sum_{\text {plaquette } j} I_{i}
$$


where $n_{j}$ is the vortex number and $I_{\Phi_{0}}$ is the current which, if circulating around a plaquette, would give an induced flux of one flux quantum $\Phi_{0}$. The last term gives a good approximation to the induced flux if, for example, the junctions are uniformly spaced around a rectangle.

If $u \neq 0$ and if the number of junctions in each plaquette is large then the phase $\phi$ dropped across each junction will be small enough that we have $\sin \phi \approx \phi$ and so Eqs. (3, tit) give us

$$
\sum_{\text {plaquette } j} I_{i} R=n_{j}-f
$$

where the 'resistance' $R$ is

$$
R=\frac{1}{2 \pi I_{c}}+\frac{1}{2(u+c) I_{\Phi_{0}}}
$$

In this approximation the energy of junction $i$ becomes $I_{i}^{2} \Phi_{0} /\left(4 \pi I_{c}\right)$ and so the total energy $U$ is

$$
U=\kappa \sum_{\text {all junctions }} I_{i}^{2}
$$

where $\kappa=\Phi_{0} /\left(4 \pi I_{c}\right)$. Since the currents are linear in $f$ the energy $U$ must be quadratic in $f$.

Now we consider a similar ladder of superconducting wires as shown in Fig. 1b. $u a$ and $c a$ are the lengths of the wires, $u$ and $c$ being integers. When the true superconducting state is established the superconducting order parameter takes the form $\psi=$ $\psi_{0} \exp \left\{i\left(\theta-\frac{2 \pi}{\Phi_{0}} \int \mathbf{A} . \mathrm{dl}\right)\right\}$. If the width of each wire is small compared with the London penetration depth then vortices cannot penetrate the wires; if in addition the width is small compared with the length then the current density $J$ will be constant:

$$
J=\frac{e\left|\psi_{0}\right|^{2} \hbar}{m}\left|\nabla \theta-\frac{2 \pi}{\Phi_{0}} \mathbf{A}\right| \equiv \frac{I}{S}
$$

where $S$ is the wire cross-sectional area and $I$ is the current carried by the wire. Integrating around a plaquette we again obtain Eq. (5) but now we have

$$
R=\frac{m a}{2 \pi e \hbar\left|\psi_{0}\right|^{2} S}+\frac{1}{2(u+c) I_{\Phi_{0}}}
$$

The total energy of the ladder is likewise given by Eq. (7) except that now $\kappa=m /\left(4 e^{2}\left|\psi_{0}\right|^{2} S\right)$.

Thus Eqs. (5) and (7) determine the solution of the currents and total energy in both the Josephson ladder and a ladder of long thin superconducting wires (and also, of course, the solution of the currents and power dissipation in a similar network of resistors and batteries). This method has already been tested [26] against Monte-Carlo calculations for a number of small Josephson networks.

Solving Eqs. (5) and (7) for a ladder of infinite length we find that the energy $U$ is

$$
\frac{U}{2 U_{0}}=\sum_{j} \varepsilon_{j}^{2}+2 \sum_{j} \sum_{k>j} \alpha^{k-j} \varepsilon_{j} \varepsilon_{k}
$$

where $\varepsilon_{j}=n_{j}-f, U_{0}=\kappa /\left(4 R^{2} \sqrt{u^{2}+2 u c}\right)$ and

$$
\alpha=1+\frac{u}{c}-\sqrt{\frac{2 u}{c}+\frac{u^{2}}{c^{2}}}
$$

$\alpha$ depends solely on $u / c$ as shown in Fig. 2; all other factors contribute only to the energy scale $U_{0}$. Note that vortices repel each other $(\alpha<1)$ and since the energy decreases exponentially with separation the vortex-vortex interaction is a short-range interaction of range $-1 / \ln \alpha$ plaquettes.

We now focus on finding the lowest energy state for any given value of $f$. For $f$ in the range $0<f<1$ we need only consider two values of $n_{j}$, namely $n_{j}=0$ and $n_{j}=1$. Hubbard [28] has shown that for particles (in our case vortices, i.e. plaquettes with $n_{j}=1$ ) interacting with a convex repulsive interaction the lowest energy state of $p$ particles in a one-dimensional chain on $q$ sites is a generalised Wigner lattice consisting of a sequence of two spacings, the spacings being the two integers which bracket $q / p$ (for example, when $q / p=7 / 3$ the two spacings will be 2 and 3 ). Fig. 3 shows the energies of these generalised Wigner lattices for various vortex densities $\rho=p / q$ as calculated by direct solution of Eq. (5). Immediately we see that there is a critical flux $f_{c}$ below which the empty ladder (i.e. $\rho=0$ ) is the lowest energy state. A more accurate numerical solution was performed for the same Josephson ladder, $\phi_{i}$ in Eq. (偣) being replaced by $\sin ^{-1}\left(I_{i} / I_{c}\right)$ instead of $I_{i} / I_{c}$; this more accurate calculation gave the transitions from one generalised Wigner lattice to another as occurring at much the same values of $f$, differing by no more than 0.01 from the values seen in Fig. 3. A ladder of larger $u$ and $c$ would have shown better agreement.

We can calculate the critical flux $f_{c}$ very simply. The energy increase $\delta U$ when one vortex is added to an empty ladder is

$$
\frac{\delta U}{2 U_{0}}=1-2 \frac{1+\alpha}{1-\alpha} f
$$

Thus $\delta U>0$ for $f<f_{c}$ where

$$
f_{c}=\frac{1-\alpha}{2(1+\alpha)}
$$

i.e. for $f<f_{c}$ the lowest energy state is the empty ladder. Furthermore we see that, as shown in Fig 2, the critical flux $f_{c}$ depends solely on the ladder ratio $u / c$; it is independent of all other parameters. Eq. (13) is consistent with the result obtained previously [24] for the square ladder with nearest neighbour interactions only.

It may look as though Fig 3 not only tells us the ground state but also the low energy excited states. However this is not the case. Excited states are not necessarily 
generalised Wigner lattices; in fact since the interaction is short range the lowest energy excitation will normally be a domain wall.

Since, for low energy states, there are only two possible values of $\varepsilon$ (i.e. $\varepsilon=-f$ and $\varepsilon=1-f$ ) Eq. (10) is reminiscent of the Ising model Hamiltonian. Let

$$
s_{j}= \begin{cases}-1 & \text { if } n_{j}=0 \\ +1 & \text { if } n_{j}=1\end{cases}
$$

Re-writing Eq. (10) in terms of these Ising variables we obtain for a ladder of $N$ plaquettes (valid in the limit $N \rightarrow \infty)$

$$
\begin{aligned}
\frac{U}{U_{0}}= & \frac{N}{2}+\frac{\left(\frac{1}{2}-f\right)^{2} N}{f_{c}} \\
& +\frac{\left(\frac{1}{2}-f\right)}{f_{c}} \sum_{j} s_{j}+\sum_{j} \sum_{k>j} \alpha^{k-j} s_{j} s_{k}
\end{aligned}
$$

This is an anti-ferromagnetic Ising model Hamiltonian in which $\frac{1}{2}-f$ plays the role of an applied field, the interaction between spins falling off exponentially with separation. The fact that at low temperatures the ladder is described by an Ising model Hamiltonian explains the Monte-Carlo result [13] (determined only for the special case $f=1 / 2$ ) that the Josephson ladder belongs to the universality class of the Ising Model.

We now explore the implications of Eq. (15). Published literature [29 31] gives no complete solution of the thermodynamics of this kind of Ising model at both nonzero field and non-zero temperature. It has been shown [32] however that the ground state of a one-dimensional Ising model with an anti-ferromagnetic interaction exhibits a complete devil's staircase [33] if the interaction is convex (i.e. decreases with separation with positive 2nd derivative); the average spin $\langle s\rangle$ per site is always a rational fraction, all rational fractions between 0 and -1 being visited as the field (in our case $\frac{1}{2}-f$ ) is increased from zero to the critical field (in our case $\frac{1}{2}-f_{c}$ ) above which the spins are all aligned opposite to the field (in our case the ladder contains no vortices).

Krantz et al [34] have calculated the precise nature of the expected devil's staircase for the case of an antiferromagnetic Ising model with an exponential interaction between spins. Using Eq. (15) their results may now be applied to the superconducting ladder. Fig. 3 shows the predicted devil's staircase for the 'square' ladder (i.e. $u=c=1$ ); note that the locations of the transition points are seen to agree with the crossing points of the energy curves. A ladder with $u<c$ would give a staircase in which steps corresponding to larger values of the denominator $q$ would be more easily resolved.

In conclusion, we have shown that in the Josephson ladder and in the superconducting wire ladder the interaction between vortices falls exponentially with separation; this is in contrast to the $2 \mathrm{D}$ array, where the logarithmic interaction between vortices leads to the Kosterlitz-Thouless phase transition [35]. At low temperatures the ladder is described by a $1 D$ Ising model (Eq. (15)) and so no phase transition is expected [36] except at zero temperature. This also leads to the ground state vortex density exhibiting a complete devil's staircase as the applied magnetic field is increased; at each step in the staircase one extra vortex penetrates the ladder. To our knowledge this is the first example of a physical system exhibiting a non-trivial devil's staircase. The fact that the devil's staircase occurs in both the Josephson ladder and the wire ladder suggests that it is a very general property of superconducting ladder structures.

In 2D networks and in wide multi-leg ladders [23] commensurate states have been observed centred on rational flux values; note that our results suggest that this is not the case for the 1D ladder, e.g. a vortex density of $\rho=1 / 5$ doesn't occur at $f=1 / 5$ but in a small region close to $f=0.3$. Likewise, the critical field $f_{c}$ has no apparent analogue in 2D. These important differences arise because in the simple $1 \mathrm{D}$ ladder the interaction is shortrange (exponential) whereas in $2 \mathrm{D}$ it is logarithmic. It is interesting that in the long range limit (i.e. small $u / c$ ) the properties of the $1 \mathrm{D}$ ladder are reminiscent of the $2 \mathrm{D}$ ladder: i.e. $f_{c} \rightarrow 0$ and the ground state vortex density $\rho=p / q \rightarrow f$.

We call for experiments to detect the predicted devil's staircase phenomenon which should be observable in low temperature mutual inductance and resistivity measurements. Each step in the staircase should give rise to a pair of metal-insulator transitions [23]: close to a step the disorder will cause high resistance while far away from any step the ordered array of vortices will cause low resistance.

From a practical point of view perhaps the most important result is that both kinds of ladder exhibit a geometry-dependent critical field $H_{g}$, analagous to $H_{c 1}$, below which all vortices are expelled. Applying Eqs. (11) and (13) one can design a ladder of any desired $H_{g}=$ $f_{c} \Phi_{0} / A$ by appropriately choosing the ladder ratio $u / c$ and the area $A$ of the elementary plaquette.

We would like to thank John Samson and Daniil Khomskii for illuminating discussions.

[1] B. J. van Wees, H. S. J. van der Zant, and J. E. Mooij Phys. Rev B35, 7291 (1987)

[2] R. Theron, J. B. Simond, J. L Galvilano, Ch. Leeman and P. Martinoli, Physica B 165-166 (1990) 1641

[3] H. S. J. van der Zant, H. A. Rijken and J. E. Mooij J. Low Temp. Phys. 82 (1991)67

[4] R. Theron J. B. Simond, Ch. Leemann, H. Beck, P. Martinoli and P. Minnhagen. Phys. Rev. Lett. 71, 1246 (1993) 
[5] S. Teitel and C. Jayaprakash Phys. Rev. Lett. 51, 1999 (1983)

[6] W. Y. Shih and D. Straud Phys. Rev. B 30, 6774 (1984)

[7] M.Y. Choi and S. Doniach Phys. Rev. B 31, 4516 (1965)

[8] P. Minnhagen Phys. Rev. B 32, 7548 (1985)

[9] E. Granato and J. M. Kosterlitz, Phys. Rev. B 33, 4767 (1986)

[10] Subodh R. Shenoy, Phys. Rev. B40 (1989) 5056

[11] U. Geigenmuller, G. Schon Physica B 165 (1990) 941

[12] D. Ariosa, A. Vallot and H. Beck J. Phys. (France) 51 (1990) 1373

[13] E. Granato, J. M. Kosterlitz, Phys. Rev. Lett. 65 (1990) 1267

[14] Ying Hong Li and S. Teitel Phys. Rev. Lett. 65, (1990) 2595

[15] H. S. J. van der Zant, F. C. Fritschy T. P. Orlando and J. E. Mooij Phys. Rev. B47, 295 (1993)

[16] A. Jonsson and P. Minnhagen Phys. Rev. B 55, 9035 (1997)

[17] J. R. Phillips, H. S. J. van der Zant, J. White and T. P. Orlando Phys. Rev. B 47, 5219 (1993)

[18] D. Straud and S. Kivelson, Phys. Rev. B35 (1987) 3478

[19] H. S. J. van der Zant, W. J. Elion, L. J. Geerligs, and J. E. Mooij Phys. Rev. B 54, 10081 (1996)

[20] H. S. J. van der Zant, M. N. Webster, J. Romijn and J. E. Mooij Phys. Rev. B 50, 340 (1994)

[21] B. Jeanneret, Ph. Fluckiger, J. L. Gavilano, Ch. Leemann and P. Martinoli, Phys, Rev B 40, 11374 (1989)

[22] M. Franz and S. Teitel, Phys. Rev. B51 6551 (1995)

[23] A. van Oudenaarden and J. E. Mooij Phys. Rev. Lett. 76, 4947, (1996); 77, 4257, (1996); Phys. Rev. B 57, 11684 (1998)

[24] H. Eikmans and J. E. van Himbergen, Phys. Rev. B 41, 8927 (1990)

[25] E. Granato, Phys. Rev. B 45, 2557 (1992)

[26] D. Khomskii and F. Kusmartsev (1991) unpublished; F. Kusmartsev, D. Farugue and D. Khomskii, Phys. Lett. A 249 (1998) 541.

[27] T.P.Orlando and K.A.Delin, 'Foundations of Applied Superconductivity', Addison-Wesley, 1991.

[28] J. Hubbard, Phys. Rev. B 17 (1978) 494.

[29] G.A.Baker, Phys.Rev. 122 (1961) 1477.

[30] J.Stephenson, Can.J.Phys. 48 (1970) 1724.

[31] R.Shrock and S.Tsai, Phys.Rev. E 55 (1997) 5184.

[32] P. Bak and R. Bruinsma, Phys. Rev. Lett. 49 (1982) 249.

[33] B.Mandelbrot, 'Fractals: Form, Chance and Dimension', Freeman, San Francisco, 1977.

[34] R.Krantz, J.Douthett and S.D.Doty, J. Math. Phys. 39 (1998) 4675-82.

[35] J. M. Kosterlitz and D. J. Thouless, J. Phys. C 6 (1973) 1181.

[36] J. M. Ziman, "Models of disorder", CUP, Cambridge, 1979.

FIG. 1. Various ladder structures: (a) the general Josephson ladder, (b) the wire ladder.

FIG. 2. The dependence of $f_{c}$ and $\alpha$ on the ladder ratio $u / c$.
FIG. 3. The solid curves show the dependence of energy per plaquette $U /\left(N U_{0}\right)$ on flux per plaquette $f$ for the 'square' ladder, i.e. $u=c=1$; each curve represents a generalised Wigner lattices of a particular vortex density $\rho=p / q$. The energies are calculated for a 512 plaquette ladder by direct solution of Eqs. (5) for the case $I_{\Phi_{0}}=\infty$. The broken line shows the predicted devil's staircase in the ground state vortex density; each visible step contains an infinite number of unresolved steps. Note the critical flux at $f=f_{c}=1 /(2 \sqrt{3}) \approx 0.289$. 
(a)

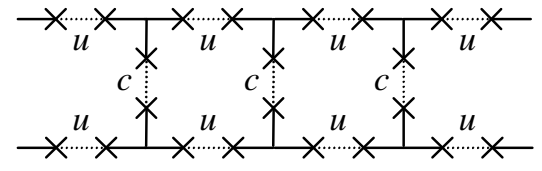

(b)

\begin{tabular}{c|c|c|c}
\hline ua & ua & ua & ua \\
$c a$ & $c a$ & $c a$ & \\
$u a$ & ua & ua & ua \\
\hline
\end{tabular}




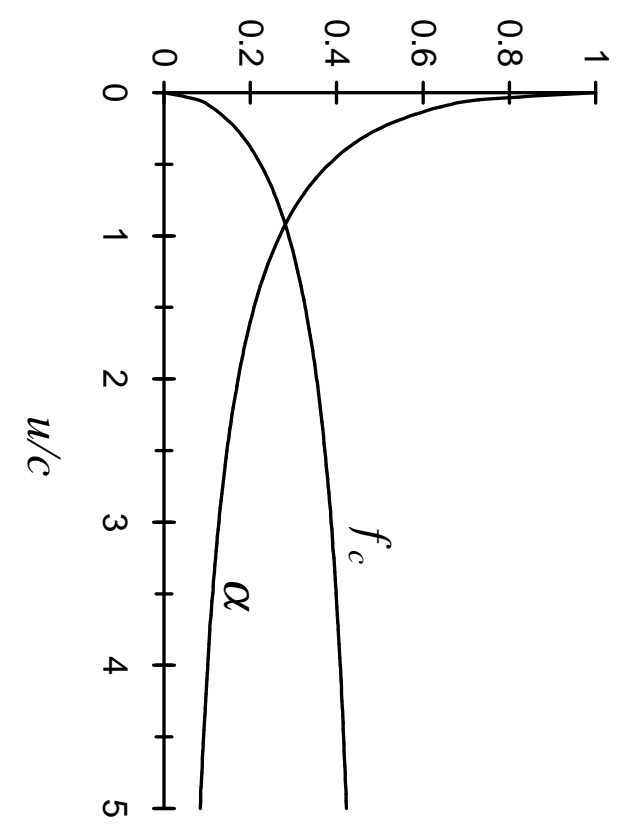




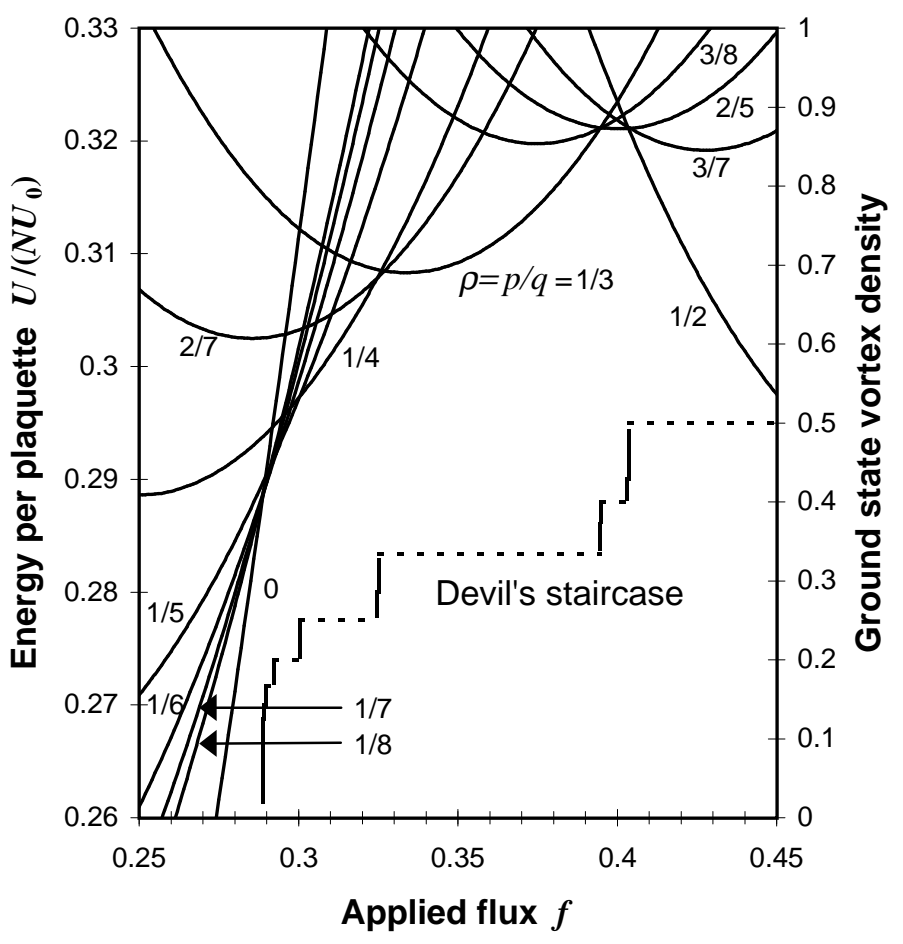

